\title{
Fuzzy Generalized Conformable Fractional Derivative
}

\author{
Atimad Harir (D), Said Melliani (D), and Lalla Saadia Chadli \\ Laboratory of Applied Mathematics and Scientific Computing, Sultan Moulay Slimane University, P.O. Box 523, \\ Beni Mellal 23000, Morocco
}

Correspondence should be addressed to Atimad Harir; atimad.harir@gmail.com

Received 14 July 2019; Revised 13 December 2019; Accepted 13 January 2020; Published 6 February 2020

Academic Editor: Ferdinando Di Martino

Copyright (C) 2020 Atimad Harir et al. This is an open access article distributed under the Creative Commons Attribution License, which permits unrestricted use, distribution, and reproduction in any medium, provided the original work is properly cited.

We give a new definition of fuzzy fractional derivative called fuzzy conformable fractional derivative. Using this definition, we prove some results and we introduce new definition of generalized fuzzy conformable fractional derivative.

\section{Introduction}

Fuzzy set theory is a powerful tool for modeling uncertainty and for processing vague or subjective information in mathematical models. Their main directions of development have been diversed, and its applications have been varied [1-4].

The derivative for fuzzy valued mappings was developed by Puri and Ralescu [5], which generalized and extended the concept of Hukuhara differentiability for set-valued mappings to the class of fuzzy mappings. Subsequently, using the H-derivative, Kaleva [6] started to develop a theory for FDE. In [7], a new well-behaved simple fractional derivative called "the conformable fractional derivative" depending just on the basic limit definition of the derivative, namely, for a function $f(0, \infty) \longrightarrow \mathbb{R}$ the (conformable) fractional derivative of order $0<q \leq 1$ of $f$ at $t>0$ was defined by

$$
T_{q} f(t)=\lim _{\varepsilon \longrightarrow 0} \frac{f\left(t+\varepsilon t^{1-q}\right)-f(t)}{\varepsilon},
$$

and is defined the fractional derivative at 0 as $\left(T_{q} f\right)(0)=\lim _{t \rightarrow 0^{+}}\left(T_{q} f\right)(t)$. The aim of this paper is to study and generalize the fuzzy conformable fractional derivative.

\section{Preliminaries}

Let us denote by $\mathbb{R}_{\mathscr{F}}=\{u: \mathbb{R} \longrightarrow[0,1]\}$ the class of fuzzy subsets of the real axis satisfying the following properties: (i) $u$ is normal, i.e., there exists an $x_{0} \in \mathbb{R}$ such that $u\left(x_{0}\right)=1$.

(ii) $u$ is fuzzy convex, i.e., for $x, y \in \mathbb{R}$ and $0<\lambda \leq 1$ :

$$
u(\lambda x+(1-\lambda) y) \geq \min [u(x), u(y)]
$$

(iii) $u$ is upper semicontinuous.

(iv) $[u]^{0}=\operatorname{cl}\{x \in \mathbb{R} \mid u(x)>0\}$ is compact.

Then, $\mathbb{R}_{\mathscr{F}}$ is called the space of fuzzy numbers. Obviously, $\mathbb{R} \subset \mathbb{R}_{\mathscr{F}}$. For $0<\alpha \leq 1$ denotes $[u]^{\alpha}=\{x \in \mathbb{R} \mid u(x) \geq \alpha\}$, then from (i) to (iv) it follows that the $\alpha$-level sets $[u]^{\alpha} \in P_{K}(\mathbb{R})$ for all $0 \leq \alpha \leq 1$ is a closed bounded interval which is denoted by $\left[u^{\alpha}\right]=\left[u_{1}^{\alpha}, u_{2}^{\alpha}\right]$. By $P_{K}(\mathbb{R})$ we denote the family of all nonempty compact convex subsets of $\mathbb{R}$ and define the addition and scalar multiplication in $P_{K}(\mathbb{R})$ as usual.

Theorem 1 (see [8]). If $u \in \mathbb{R}_{\mathscr{F}}$, then

(i) $[u]^{\alpha} \in P_{K}(\mathbb{R})$ for all $0 \leq \alpha \leq 1$.

(ii) $[u]^{\alpha_{2}} \subset[u]^{\alpha_{1}}$ for all $0 \leq \alpha_{1} \leq \alpha_{2} \leq 1$.

(iii) $\left\{\alpha_{k}\right\} \subset[0,1]$ is a nondecreasing sequence which converges to $\alpha$, then

$$
[u]^{\alpha}=\bigcap_{k \geq 1}[u]^{\alpha_{k}}
$$

Conversely, if $A_{\alpha}=\left\{\left[u_{1}^{\alpha}, u_{2}^{\alpha}\right] ; \alpha \in(0,1]\right\}$ is a family of closed real intervals verifying (i) and (ii), then $\left\{A_{\alpha}\right\}$ defined $a$ 
fuzzy number $u \in \mathbb{R}_{\mathscr{F}}$ such that $[u]^{\alpha}=A_{\alpha}$ for $0<\alpha \leq 1$ and $[u]^{0}=\overline{\mathrm{U}_{0<\alpha \leq 1} A_{\alpha}} \subset A_{0}$.

Lemma 1. Let $u, v: X \longrightarrow[0,1]$ be the fuzzy sets. ([9]).

Then, $u=v$ if and only if $[u]^{\alpha}=[v]^{\alpha}$ for all $\alpha \in[0,1]$ see

The following arithmetic operations on fuzzy numbers are well known and frequently used below. If $u, v \in \mathbb{R}_{\mathscr{F}}$, then

$$
\begin{aligned}
{[u+v]^{\alpha} } & =\left[u_{1}^{\alpha}+v_{1}^{\alpha}, u_{2}^{\alpha}+v_{2}^{\alpha}\right], \\
& {[\lambda u]^{\alpha}=\lambda[u]^{\alpha}= \begin{cases}{\left[\lambda u_{1}^{\alpha}, \lambda u_{2}^{\alpha}\right],} & \lambda \geq 0, \\
{\left[\lambda u_{2}^{\alpha}, \lambda u_{1}^{\alpha}\right],} & \lambda<0 .\end{cases} }
\end{aligned}
$$

Definition 1. Let $u, v \in \mathbb{R}_{\mathscr{F}}$. If there exists $w \in \mathbb{R}_{\mathscr{F}}$ such that $u=v+w$, then $w$ is called the $H$-difference of $u, v$ and it is denoted $u \ominus v$.

Theorem 2 (see [10])

(i) Let us denote

$$
\overline{0}= \begin{cases}1, & t=0, \\ 0, & t \neq 0 .\end{cases}
$$

Then, $\overline{0} \in \mathbb{R}_{\mathscr{F}}$ be a neutral element with respect to + , i.e., $u+\overline{0}=\overline{0}+u \quad u \in \mathbb{R}_{\mathscr{F}}$ :

(i) With respect to $\overline{0}$, none of $u \in \mathbb{R}_{\mathscr{F}} / \mathbb{R}$ has opposite in $\mathbb{R}_{\mathscr{F}}$.

(ii) For any $a, b \in \mathbb{R}$ with $a, b \geq 0$ or $a, b \leq 0$ and any $u \in \mathbb{R}_{\mathscr{F}}$, we have $(a+b) \cdot u=a \cdot u+b \cdot u$, for general $a, b \in \mathbb{R}$ the above property does not hold.

(iii) For any $\lambda \in \mathbb{R}$ and any $u, v \in \mathbb{R}_{\mathscr{F}}$, we have $\lambda \cdot(u+v)=\lambda \cdot u+\lambda \cdot v$.

(iv) For any $\lambda, v \in \mathbb{R}$ and any $u \in \mathbb{R}_{\mathscr{F}}$, we have $\lambda \cdot(\nu \cdot u)=(\lambda \cdot \nu) \cdot u$.

Define $d: \mathbb{R}_{\mathscr{F}} \times \mathbb{R}_{\mathscr{F}} \longrightarrow \mathbb{R}_{+} \cup\{0\}$ by the following equation:

$$
d(u, v)=\sup _{\alpha \in[0,1]} d_{H}\left([u]^{\alpha},[v]^{\alpha}\right), \quad \text { for all } u, v \in \mathbb{R}_{\mathscr{F}},
$$

where $d_{H}$ is the Hausdorff metric:

$$
d_{H}\left([u]^{\alpha},[v]^{\alpha}\right)=\max \left\{|| u_{1}^{\alpha}-v_{1}^{\alpha}|,| u_{2}^{\alpha}-v_{2}^{\alpha} \|\right\} .
$$

It is well known that $\left(\mathbb{R}_{\mathscr{F}}, d\right)$ is a complete metric space. We list the following properties of $d(u, v)$ :

$$
\begin{aligned}
& d(u+w, v+w)=d(u, v), \\
& d(u, v)=d(v, u), \\
& d(k u, k v)=|k| d(u, v), \\
& d(u, v) \leq d(u, w)+d(w, v),
\end{aligned}
$$

for all $u, v, w \in \mathbb{R}_{\mathscr{F}}$ and $\lambda \in \mathbb{R}$.

Let $\left(A_{k}\right)$ be a sequence in $P_{K}(\mathbb{R})$ converging to $A$. Then, theorem in [6] gives us an expression for the limit.
Theorem 3 (see [6]). If $d\left(A_{k}, A\right) \longrightarrow 0$ as $k \longrightarrow \infty$, then

$$
A=\bigcap_{k \geq 1} \overline{\bigcup_{m \geq k} A_{m}}
$$

Let $I=(0, a) \subset \mathbb{R}$ be an interval. We denote by $C\left(I, \mathbb{R}_{\mathscr{F}}\right)$ that the space of all continuous fuzzy functions on $I$ is a complete metric space with respect to the metric:

$$
h(u, v)=\sup _{t \in I} d(u(t), v(t)) .
$$

\section{The Fuzzy Conformable Fractional Differentiability}

Definition 2. Let $F: I \longrightarrow \mathbb{R}_{\mathscr{F}}$ be a fuzzy function. qth order "fuzzy conformable fractional derivative" of $\mathrm{F}$ is defined by

$$
\begin{aligned}
T_{q}(F)(t) & =\lim _{\varepsilon \longrightarrow 0^{+}} \frac{F\left(t+\varepsilon t^{1-q}\right) \ominus F(t)}{\varepsilon} \\
& =\lim _{\varepsilon \longrightarrow 0^{+}} \frac{F(t) \ominus F\left(t-\varepsilon t^{1-q}\right)}{\varepsilon},
\end{aligned}
$$

for all $t>0$ and $q \in(0,1)$. Let $F^{(q)}(t)$ stands for $T_{q}(F)(t)$. Hence,

$$
F^{(q)}(t)=\lim _{\varepsilon \longrightarrow 0^{+}} \frac{F\left(t+\varepsilon t^{1-q}\right) \ominus F(t)}{\varepsilon}=\lim _{\varepsilon \longrightarrow 0^{+}} \frac{F(t) \ominus F\left(t-\varepsilon t^{1-q}\right)}{\varepsilon} .
$$

If $F$ is $q$-differentiable in some $(0, a)$ and $\lim _{t \longrightarrow 0^{+}} F^{(q)}(t)$,

$$
F^{(q)}(0)=\lim _{t \longrightarrow 0^{+}} F^{(q)}(t),
$$

and the limits exist (in the metric $d$ ).

Remark 1. From the definition, it directly follows that if $F$ is $q$-differentiable then the multivalued mapping $F_{\alpha}$ is q-differentiable for all $\alpha \in[0,1]$ and

$$
T_{q} F_{\alpha}=\left[F^{(q)}(t)\right]^{\alpha}
$$

where $T_{q} F_{\alpha}$ is denoted from the conformable fractional derivative of $F_{\alpha}$ of order $q$. The converse result does not hold, since the existence of Hukuhara difference $[x]^{\alpha} \ominus[y]^{\alpha}$, $\alpha \in[0,1]$, does not imply the existence of $H$-difference $x \ominus y$.

However, for the converse result we have the following.

Theorem 4. Let $F: I \longrightarrow \mathbb{R}_{\mathscr{F}}$ satisfy the assumptions:

(i) For each $t \in I$ there exists a $\beta>0$ such that the $H$-difference $F\left(t+\varepsilon t^{1-q}\right) \ominus F(t) \quad$ and $F(t) \ominus F\left(t-\varepsilon t^{1-q}\right)$ exists for all $0 \leq \varepsilon<\beta$.

(ii) The set-valued mappings $F_{\alpha}, \alpha \in[0,1]$ are uniformly Hukuhara conformable fractional derivative of order $q$ with derivative $T_{q} F_{\alpha}$, i.e., for each $t \in I$ and $h>0$ there exists a $\delta>0$ such that 


$$
\begin{aligned}
& d_{H}\left(\frac{\left(F_{\alpha}\left(t+\varepsilon t^{1-q}\right)-F_{\alpha}(t)\right)}{\varepsilon, T_{q} F_{\alpha}(t)}\right)<h, \\
& d_{H}\left(\frac{\left(F_{\alpha}(t)-F_{\alpha}\left(t-\varepsilon t^{1-q}\right)\right)}{\varepsilon, T_{q} F_{\alpha}(t)}\right)<h,
\end{aligned}
$$

for all $0 \leq \varepsilon<\delta$ and $\alpha \in[0,1]$. Then, $F$ is q-differentiable, and the derivative is given by (14).

Proof. Consider the family $\left\{T_{q} F_{\alpha} \mid \alpha \in[0,1]\right\}$ By definition $T_{q} F_{\alpha}(t)$ is a compact, convex, and nonempty subset of $\mathbb{R}$.
If $\alpha_{1} \leq \alpha_{2}$, then by assumption $(i)$,

$F_{\alpha_{1}}\left(t+\varepsilon t^{1-q}\right)-F_{\alpha_{1}}(t) \supset F_{\alpha_{2}}\left(t+\varepsilon t^{1-q}\right)-F_{\alpha_{2}}(t), \quad$ for $0 \leq \varepsilon<\beta$,

and consequently

$$
T_{q} F_{\alpha_{1}} \supset T_{q} F_{\alpha_{2}}
$$

Let $\alpha>0$ and $\alpha_{k}$ be a nondecreasing sequence converging to $\alpha$. For $h>0$ choose $\varepsilon>0$ such that equation(15) holds true. Then, the triangle inequality yields

$$
\begin{aligned}
d_{H}\left(T_{q} F_{\alpha}(t), T_{q} F_{\alpha_{k}}(t)\right) \leq & d_{H}\left(T_{q} F_{\alpha}(t), \frac{\left(F_{\alpha}\left(t+\varepsilon t^{1-q}\right)-F_{\alpha}(t)\right)}{\varepsilon}\right)+d_{H}\left(\frac{\left(F_{\alpha}\left(t+\varepsilon t^{1-q}\right)-F_{\alpha}(t)\right)}{\varepsilon}, \frac{\left(F_{\alpha_{k}}\left(t+\varepsilon t^{1-q}\right)-F_{\alpha_{k}}(t)\right)}{\varepsilon}\right) \\
& +d_{H}\left(\frac{\left(F_{\alpha_{k}}\left(t+\varepsilon t^{1-q}\right)-F_{\alpha_{k}}(t)\right)}{\varepsilon}, T_{q} F_{\alpha_{k}}(t)\right) \leq 2 h+d_{H} \\
& \cdot\left(\frac{\left(F_{\alpha}\left(t+\varepsilon t^{1-q}\right)-F_{\alpha}(t)\right)}{\varepsilon}, \frac{\left(F_{\alpha_{k}}\left(t+\varepsilon t^{1-q}\right)-F_{\alpha_{k}}(t)\right)}{\varepsilon}\right) .
\end{aligned}
$$

By assumption (i), the rightmost term goes to zero as $k \longrightarrow \infty$ and hence

$$
\lim _{k \longrightarrow \infty} d_{H}\left(T_{q} F_{\alpha}(t), T_{q} F_{\alpha_{k}}(t)\right)=0 .
$$

Now by Theorem 3 and (18) we have

$$
T_{q} F_{\alpha}(t)=\bigcup_{k \geq 1} T_{q} F_{\alpha_{k}}(t) .
$$

If $\alpha=0$, then using equation (3.1) in [11], we deduce as before that

$$
\lim _{k \longrightarrow \infty} d_{H}\left(T_{q} F_{0}(t), T_{q} F_{\alpha_{k}}(t)\right)=0,
$$

where $\left(\alpha_{k}\right)$ is a nonincreasing sequence converging to zero and consequently

$$
T_{q} F_{0}(t)=\overline{\bigcup_{k \geq 1} T_{q} F_{\alpha_{k}}}
$$

Then, from Theorem 1 it follows that there is an element $u \in \mathbb{R}_{\mathscr{F}}$ such that

$$
[u]^{\alpha}=T_{q} F_{\alpha}(t), \quad \text { for } \alpha \in[0,1] .
$$

Furthermore, let $t \in I, h>0$, and $\delta>0$ be as in (ii). Then,

$$
d\left(\frac{\left(F\left(t+\varepsilon t^{1-q}\right) \ominus F(t)\right)}{\varepsilon}, u\right)=\sup _{0<\alpha \leq 1} d_{H}\left(\frac{\left(F_{\alpha}\left(t+\varepsilon t^{1-q}\right)-F_{\alpha}(t)\right)}{\varepsilon, T_{q} F_{\alpha}(t)}\right)<h
$$

for all $0 \leq \varepsilon<\delta$ and similarly for $d\left(\left(F\left(t+\varepsilon t^{1-q}\right) \ominus F(t)\right) / \varepsilon, u\right)$. Hence, $F^{(q)}(t)=u$ has the theorem.

$$
\left[F^{(q)}(t)\right]^{\alpha}=\left[\left(f_{1}^{\alpha}\right)^{(q)}(t),\left(f_{2}^{\alpha}\right)^{(q)}(t)\right]
$$

Theorem 5. Let $F: I \longrightarrow \mathbb{R}_{\mathscr{F}}$ be q-differentiable. Denote $F_{\alpha}(t)=\left[f_{1}^{\alpha}(t), f_{2}^{\alpha}(t)\right], \alpha \in[0,1]$. Then, $f_{1}^{\alpha}(t)$ and $f_{2}^{\alpha}(t)$ are Proof. If $\varepsilon>0$ and $\alpha \in[0,1]$, we have $q$-differentiable and

$$
\left[F\left(t+\varepsilon t^{1-q}\right) \ominus F(t)\right]^{\alpha}=\left[f_{1}^{\alpha}\left(t+\varepsilon t^{1-q}\right)-f_{1}^{\alpha}(t), f_{2}^{\alpha}\left(t+\varepsilon t^{1-q}\right)-f_{2}^{\alpha}(t)\right] .
$$


Dividing by $\varepsilon$, we have

$$
\frac{\left[F\left(t+\varepsilon t^{1-q}\right) \ominus F(t)\right]^{\alpha}}{\varepsilon}=\left[\frac{f_{1}^{\alpha}\left(t+\varepsilon t^{1-q}\right)-f_{1}^{\alpha}(t)}{\varepsilon}, \frac{f_{2}^{\alpha}\left(t+\varepsilon t^{1-q}\right)-f_{2}^{\alpha}(t)}{\varepsilon}\right] .
$$

Similarly, we obtain

$$
\frac{\left[F(t) \ominus F\left(t-\varepsilon t^{1-q}\right)\right]^{\alpha}}{\varepsilon}=\left[\frac{f_{1}^{\alpha}(t)-f_{1}^{\alpha}\left(t-\varepsilon t^{1-q}\right)}{\varepsilon}, \frac{f_{2}^{\alpha}(t)-f_{2}^{\alpha}\left(t-\varepsilon t^{1-q}\right)}{\varepsilon}\right],
$$

and passing to the limit gives the theorem.

Note that this definition and theorem of conformable fractional derivative are very restrictive; for instance, if $F(t)=c \cdot g(t)$, where $c$ is a fuzzy number and $g:[a, b] \longrightarrow \mathbb{R}^{+}$is a function and is $q$-differentiable for some $q \in(0,1]$ with $g^{(q)}(t)<0$, then $F$ is not $q$-differentiable. To avoid this difficulty, we introduce a more general definition of the conformable fractional derivative for fuzzynumber-valued function.

\section{The Generalized Fuzzy Conformable Fractional Differentiability}

We consider the following definition.

Definition 3. Let $F: I \longrightarrow \mathbb{R}_{\mathscr{F}}$ be a fuzzy function and $q \in(0,1]$. One says, $F$ is $q_{(1)}$-differentiable at point $t>0$ if there exists an element $F^{(q)}(t) \in \mathbb{R}_{\mathscr{F}}$ such that for all $\varepsilon>0$ sufficiently near to 0 there exist $F\left(t+\varepsilon t^{1-q}\right) \ominus F(t)$, $F(t) \ominus F\left(t-\varepsilon t^{1-q}\right)$, and the limits (in the metric $d$ ):

$$
\lim _{\varepsilon \longrightarrow 0^{+}} \frac{F\left(t+\varepsilon t^{1-q}\right) \ominus F(t)}{\varepsilon}=\lim _{\varepsilon \longrightarrow 0^{+}} \frac{F(t) \ominus F\left(t-\varepsilon t^{1-q}\right)}{\varepsilon}=F^{(q)}(t),
$$

where $F$ is $q_{(2)}$-differentiable at $t>0$ if for all $\varepsilon<0$ sufficiently near to 0 , then there exist $F\left(t+\varepsilon t^{1-q}\right) \ominus F(t)$ and $F(t) \ominus F\left(t-\varepsilon t^{1-q}\right)$ :

$$
\lim _{\varepsilon \longrightarrow 0^{-}} \frac{F\left(t+\varepsilon t^{1-q}\right) \ominus F(t)}{\varepsilon}=\lim _{\varepsilon \longrightarrow 0^{-}} \frac{F(t) \ominus F\left(t-\varepsilon t^{1-q}\right)}{\varepsilon}=F^{(q)}(t) .
$$

If $F$ is $q_{(n)}$-differentiable at $t>0$, we denote its $q$-derivatives $(q \in(0,1])$ by $F_{n}^{(q)}(t)$, for $n=1,2$.

Example 1. Let $g: I \longrightarrow \mathbb{R}^{+}$and define $F: I \longrightarrow \mathbb{R}_{\mathscr{F}}$ by $F(t)=c \cdot g(t)$ for all $t \in I$, where $c$ is the fuzzy number. If $g$ is $q$-differentiable at $t_{0} \in I$, then $F$ is the generalized fuzzy conformable fractional derivative at $t_{0} \in I$ and we have
$F^{(q)}\left(t_{0}\right)=c \cdot g^{(q)}\left(t_{0}\right)$. For instance, if $g^{(q)}\left(t_{0}\right)>0, \quad F$ is $q_{(1)}$-differentiable. If $g^{(q)}\left(t_{0}\right)<0$, then $F$ is $q_{(2)^{-}}$ differentiable.

Remark 2. In the previous definition, $q_{(1)}$-differentiable corresponds to Definition 3, so this differentiability concept is a generalization of Definition 2 and obviously more general. For instance, in the previous example, for $F(t)=$ $c \cdot g(t)$ with $g^{(q)}\left(t_{0}\right)<0$, we have. $F^{(q)}\left(t_{0}\right)=c \cdot g^{(q)}\left(t_{0}\right)$

Theorem 6. Let $F: I \longrightarrow \mathbb{R}_{\mathscr{F}}$ be fuzzy function, where $F_{\alpha}(t)=\left[f_{1}^{\alpha}(t), f_{2}^{\alpha}(t)\right], \alpha \in[0,1]:$

(i) If $F$ is $q_{(1)}$-differentiable, then $f_{1}^{\alpha}(t)$ and $f_{2}^{\alpha}(t)$ are q-differentiable and

$$
\left[F^{\left(q_{(1)}\right)}(t)\right]^{\alpha}=\left[\left(f_{1}^{\alpha}\right)^{(q)}(t),\left(f_{2}^{\alpha}\right)^{(q)}(t)\right]
$$

(ii) If $F$ is $q_{(2)}$-differentiable, then $f_{1}^{\alpha}(t)$ and $f_{2}^{\alpha}(t)$ are q-differentiable and

$$
\left[F^{\left(q_{(2)}\right)}(t)\right]^{\alpha}=\left[\left(f_{2}^{\alpha}\right)^{(q)}(t),\left(f_{1}^{\alpha}\right)^{(q)}(t)\right] .
$$

Proof

(i) See demonstration of Theorem 5

(ii) If $\varepsilon<0, q \in(0,1]$, and $\alpha \in[0,1]$, then we have

$$
\begin{aligned}
{\left[F\left(t+\varepsilon t^{1-q}\right) \ominus F(t)\right]^{\alpha}=} & {\left[f_{1}^{\alpha}\left(t+\varepsilon t^{1-q}\right)-f_{1}^{\alpha}(t), f_{2}^{\alpha}\left(t+\varepsilon t^{1-q}\right)\right.} \\
& \left.-f_{2}^{\alpha}(t)\right] .
\end{aligned}
$$

and multiplying by $1 / \mathcal{E}$, we have 


$$
\frac{\left[F\left(t+\varepsilon t^{1-q}\right) \ominus F(t)\right]^{\alpha}}{\varepsilon}=\left[\frac{f_{2}^{\alpha}\left(t+\varepsilon t^{1-q}\right)-f_{2}^{\alpha}(t)}{\varepsilon}, \frac{f_{1}^{\alpha}\left(t+\varepsilon t^{1-q}\right)-f_{1}^{\alpha}(t)}{\varepsilon}\right] .
$$

Similarly, we obtain

$$
\frac{\left[F(t) \ominus F\left(t-\varepsilon t^{1-q}\right)\right]^{\alpha}}{\varepsilon}=\left[\frac{f_{2}^{\alpha}(t)-f_{2}^{\alpha}\left(t-\varepsilon t^{1-q}\right)}{\varepsilon}, \frac{f_{1}^{\alpha}(t)-f_{1}^{\alpha}\left(t-\varepsilon t^{1-q}\right)}{\varepsilon}\right],
$$

and passing to the limit we have $\left[F^{\left(q_{(2)}\right)}(t)\right]^{\alpha}=\left[\left(f_{2}^{\alpha}\right)^{(q)}(t)\right.$, $\left.\left(f_{1}^{\alpha}\right)^{(q)}(t)\right]$.

Theorem 7. Let $F: I \longrightarrow \mathbb{R}_{\mathscr{F}}$ and $g: I \longrightarrow \mathbb{R}$ be two $q$-differentiable functions at a point $t>0$ ( $F$ is the generalized fuzzy conformable fractional derivative Definition 3 and $q \in(0,1])$ :

(i) If $g(t) \cdot T_{q}(g)(t)>0$ and $F$ is $q_{(1)}$-differentiable, then $g \cdot F$ is $q_{(1)}$-differentiable and

$T_{q}(g \cdot F)(t)=g(t) \cdot T_{q}(F)(t)+F(t) \cdot T_{q}(g)(t)$.

(ii) If $g(t) \cdot T_{q}(g)(t)<0$ and $F$ is $q_{(2)}$-differentiable, then $g \cdot F$ is $q_{(2)}$-differentiable and

$T_{q}(g \cdot F)(t)=g(t) \cdot T_{q}(F)(t)+F(t) \cdot T_{q}(g)(t)$.
Proof. We present the details only for the case $(i)$, since the other case is analogous. $F$ is $q_{(1)}$-differentiable at $t>0$ the $H$ difference $F(t) \ominus F\left(t+\varepsilon t^{1-q}\right)$ exists for $\varepsilon>0$ sufficiently small, i.e., there exists $u_{1}\left(t, \varepsilon t^{1-q}\right) \in \mathbb{R}_{\mathscr{F}}$, such that

$$
F(t)=F\left(t+\varepsilon t^{1-q}\right)+u_{1}\left(t, \varepsilon t^{1-q}\right) \text {. }
$$

Also,

$$
g(t)=g\left(t+\varepsilon t^{1-q}\right)+u_{2}\left(t, \varepsilon t^{1-q}\right)
$$

where $u_{2}\left(t, \varepsilon t^{1-q}\right)=g(t)-g\left(t+\varepsilon t^{1-q}\right)$ for $\varepsilon>0$ sufficiently small.

By Theorem 2, we obtain

$$
\begin{aligned}
F(t) \cdot g(t)= & F\left(t+\varepsilon t^{1-q}\right) \cdot g\left(t+\varepsilon t^{1-q}\right)+F\left(t+\varepsilon t^{1-q}\right) \\
& \cdot u_{2}\left(t, \varepsilon t^{1-q}\right)+g\left(t+\varepsilon t^{1-q}\right) \cdot u_{1}\left(t, \varepsilon t^{1-q}\right) \\
& +u_{1}\left(t, \varepsilon t^{1-q}\right) \cdot u_{2}\left(t, \varepsilon t^{1-q}\right),
\end{aligned}
$$

that is, the $H$-difference $F(t) \cdot g(t) \ominus F\left(t+\varepsilon t^{1-q}\right)$. $g\left(t+\varepsilon t^{1-q}\right)$ exists and we have

$$
F(t) \cdot g(t) \ominus F\left(t+\varepsilon t^{1-q}\right) \cdot g\left(t+\varepsilon t^{1-q}\right)=F\left(t+\varepsilon t^{1-q}\right) \cdot u_{2}\left(t, \varepsilon t^{1-q}\right)+g\left(t+\varepsilon t^{1-q}\right) \cdot u_{1}\left(t, \varepsilon t^{1-q}\right)+u_{1}\left(t, \varepsilon t^{1-q}\right) \cdot u_{2}\left(t, \varepsilon t^{1-q}\right) .
$$

Multiplying with $1 / \varepsilon$ and passing to limit with $\varepsilon>0$, by Proof of Lemma 3.2 [12], we obtain

$$
\begin{aligned}
& \lim _{\varepsilon \longrightarrow 0^{+}} \frac{F(t) \cdot g(t) \ominus F\left(t+\varepsilon t^{1-q}\right) \cdot g\left(t+\varepsilon t^{1-q}\right)}{\varepsilon}=\lim _{\varepsilon \longrightarrow 0^{+}} F\left(t+\varepsilon t^{1-q}\right) \cdot \frac{u_{2}\left(t, \varepsilon t^{1-q}\right)}{\varepsilon} \\
& +\lim _{\varepsilon \longrightarrow 0^{+}} g\left(t+\varepsilon t^{1-q}\right) \cdot \frac{u_{1}\left(t, \varepsilon t^{1-q}\right)}{\varepsilon}+\lim _{\varepsilon \longrightarrow 0^{+}} \frac{u_{1}\left(t, \varepsilon t^{1-q}\right)}{\varepsilon} \cdot u_{2}\left(t, \varepsilon t^{1-q}\right), \\
& \lim _{\varepsilon \longrightarrow 0^{+}} \frac{F(t) \cdot g(t) \ominus F\left(t+\varepsilon t^{1-q}\right) \cdot g\left(t+\varepsilon t^{1-q}\right)}{\varepsilon}=F(t) \cdot T_{q} g(t)+g(t) \cdot T_{q} F(t)+T_{q} g(t) \cdot \lim _{\varepsilon \longrightarrow 0^{+}} u_{2}\left(t, \varepsilon t^{1-q}\right) \\
& =F(t) \cdot T_{q} g(t)+g(t) \cdot T_{q} F(t) .
\end{aligned}
$$


Analogously, we obtain

$$
\lim _{\varepsilon \longrightarrow 0^{+}} \frac{F(t) \cdot g(t) \ominus F\left(t-\varepsilon t^{1-q}\right) \cdot g\left(t-\varepsilon t^{1-q}\right)}{\varepsilon}=F(t) \cdot T_{q} g(t)+g(t) \cdot T_{q} F(t),
$$

and the conclusion in the case $(i)$ is obtained.

$$
T_{q_{(2)}} F(t)=t^{1-q} D_{2}^{1} F(t) .
$$

Theorem 8. Let $q \in(0,1]$ :

(i) If $F$ is (1)-differentiable and $F$ is $q_{(1)}$-differentiable, then

$$
T_{q_{(1)}} F(t)=t^{1-q} D_{1}^{1} F(t) .
$$

(ii) If $F$ is (2)-differentiable and $F$ is $q_{(2)}$-differentiable, then
Note that the definition of $(n)$-differentiable or $\left(D_{n}^{1}\right)$ for $n \in 1,2$, see $[12-14]$.

Proof. We present the details only for Case (i), since the other case is analogous. Let $h=\varepsilon t^{1-q}$ in Definition 7, and then $\varepsilon=t^{q-1} h$. Therefore, if $\varepsilon>0$ and $\alpha \in[0,1]$, we have

$$
\left[F\left(t+\varepsilon t^{1-q}\right) \ominus F(t)\right]^{\alpha}=\left[f_{1}^{\alpha}\left(t+\varepsilon t^{1-q}\right)-f_{1}^{\alpha}(t), f_{2}^{\alpha}\left(t+\varepsilon t^{1-q}\right)-f_{2}^{\alpha}(t)\right] .
$$

Dividing by $\varepsilon$, we have

$$
\frac{\left[F\left(t+\varepsilon t^{1-q}\right) \ominus F(t)\right]^{\alpha}}{\varepsilon}=\left[\frac{f_{1}^{\alpha}\left(t+\varepsilon t^{1-q}\right)-f_{1}^{\alpha}(t)}{\varepsilon}, \frac{f_{2}^{\alpha}\left(t+\varepsilon t^{1-q}\right)-f_{2}^{\alpha}(t)}{\varepsilon}\right],
$$

and passing to the limit,

$$
\begin{aligned}
\lim _{\varepsilon \longrightarrow 0^{+}} \frac{\left[F\left(t+\varepsilon t^{1-q}\right) \ominus F(t)\right]^{\alpha}}{\varepsilon} & =\lim _{\varepsilon \longrightarrow 0^{+}}\left[\frac{f_{1}^{\alpha}\left(t+\varepsilon t^{1-q}\right)-f_{1}^{\alpha}(t)}{\varepsilon}, \frac{f_{2}^{\alpha}\left(t+\varepsilon t^{1-q}\right)-f_{2}^{\alpha}(t)}{\varepsilon}\right] \\
& =\lim _{h \longrightarrow 0^{+}}\left[\frac{f_{1}^{\alpha}(t+h)-f_{1}^{\alpha}(t)}{t^{q-1} h}, \frac{f_{2}^{\alpha}(t+h)-f_{2}^{\alpha}(t)}{t^{q^{-1}} h}\right] \\
& =t^{1-q} \lim _{h \longrightarrow 0^{+}}\left[\frac{f_{1}^{\alpha}(t+h)-f_{1}^{\alpha}(t)}{h}, \frac{f_{2}^{\alpha}(t+h)-f_{2}^{\alpha}(t)}{h}\right] \\
& =t^{1-q}\left[\left(f_{1}^{\alpha}\right)^{\prime}(t),\left(f_{2}^{\alpha}\right)^{\prime}(t)\right] .
\end{aligned}
$$

Similarly, we obtain

$$
\frac{\left[F(t) \ominus F\left(t-\varepsilon t^{1-q}\right)\right]^{\alpha}}{\varepsilon}=\left[\frac{f_{1}^{\alpha}(t)-f_{1}^{\alpha}\left(t-\varepsilon t^{1-q}\right)}{\varepsilon}, \frac{f_{2}^{\alpha}(t)-f_{2}^{\alpha}\left(t-\varepsilon t^{1-q}\right)}{\varepsilon}\right],
$$

and passing to the limit and $\varepsilon=t^{q^{-1}} h$ gives $T_{q_{(1)}} F(t)=$ $t^{1-q}\left[\left(f_{1}^{\alpha}\right)^{\prime}(t),\left(f_{2}^{\alpha}\right)^{\prime}(t)\right]$.

Example 2. Let $c \in \mathbb{R}_{\mathscr{F}}$ and $g(0, a) \longrightarrow \mathbb{R}$, if $g$ is $q$ differentiable on $(0, a)$, then the function $F(0, a) \longrightarrow \mathbb{R}$ defined by $F(t)=c \cdot g(t), t \in(0, a)$. Then, $\mathrm{F}$ is $q_{i}$-differentiable, for $i=1,2$, and we have $F^{q_{(i)}}(t)=c \cdot g^{q_{(i)}}(t)$ for $i=1,2$. $c=(1,2,3)$ and. $[c]^{\alpha}=[1+\alpha, 3-\alpha]$.

(i) Let $g(t)=e^{t}$, so $F(t)=(1,2,3) e^{t}$ and $T_{q}\left(e^{t}\right)=$ $t^{1-q} e^{t}$, then it is easy to see that $T_{q_{(1)}} F(t)=$ $(1,2,3) t^{1-q} e^{t}$, i.e.,

$$
\left[F^{q_{(1)}}(t)\right]^{\alpha}=\left[(1+\alpha) t^{1-q} e^{t},(3-\alpha) t^{1-q} e^{t}\right] .
$$


(ii) Let $g(t)=e^{-t}$, so $F(t)=(1,2,3) e^{-t}$ and $T_{q}\left(e^{-t}\right)=$ $-t^{1-q} e^{-t}$, then it is easy to see that $T_{q_{(2)}} F(t)=$ $-(1,2,3) t^{1-q} e^{-t}$, i.e.,

$$
\left[F^{q_{(2)}}(t)\right]^{\alpha}=\left[(-3+\alpha) t^{1-q} e^{-t},(-1-\alpha) t^{1-q} e^{-t}\right] .
$$

\section{Conclusion}

We have investigated generalized fuzzy conformable fractional differentiability. The conformable $q$-differentiability introduced here is a very general differentiability concept, being also practically applicable, and we can calculate by the fuzzy conformable derivative of the product of two functions $\left(T_{q}(f \cdot g)\right)$ because all fractional derivatives do not satisfy the known formula $T_{q}(f \cdot g)=T_{q}(f) g+f T_{q}(g)$.

The disadvantage of fuzzy generalized conformable differentiability of a function seems to be that a simple fuzzy differential equation $\left(y^{(q)}+y=0,0<q \leq 1, y(0)=\right.$ $y_{0} \in \mathbb{R}_{\mathscr{F}}$ ) has not got a unique solution, so it may have several solutions. The advantage of the existence of these solutions is that we can choose the solution that reflects better the behaviour of the modelled real-world system.

For further research we propose the study for fuzzy fractional differential equations, by using the generalized conformable differentiability concept. In addition, we propose to extend the results of the present paper and to combine them with the results in [15] for fuzzy fractional differential equations.

\section{Data Availability}

No data were used to support this study.

\section{Conflicts of Interest}

The authors declare that they have no conflicts of interest.

\section{References}

[1] M. Senol, O. Tasbozan, and A. Kurt, "Numerical solutions of fractional Burgers' type equations with conformable derivative," Chinese Journal of Physics, vol. 58, pp. 75-84, 2019.

[2] O. Özkan and A. Kurt, "Exact solutions of fractional partial differential equation systems with conformable derivative," Filomat, vol. 33, no. 5, pp. 1313-1322, 2019.

[3] M. Senol, S. Atpinar, Z. Zararsiz, S. Salahshour, and A. Ahmadian, "Approximate solution of time-fractional fuzzy partial differential equations," Computational and Applied Mathematics, vol. 38, no. 1, p. 18, 2019.

[4] M. Sengonul and Z. Zararsiz, "Some additions to the fuzzy convergent and fuzzy bounded sequences spaces of fuzzy numbers," Abstract and Applied Analysis, vol. 2011, Article ID 837584, 12 pages, 2011.

[5] M. Puri and D. Ralescu, "Differential and fuzzy functions," Journal of Mathematical Analysis and Applications, vol. 91, no. 2, pp. 552-558, 1983.

[6] O. Kaleva, "Fuzzy differential equations," Fuzzy Sets and Systems, vol. 24, no. 3, pp. 301-317, 1987.
[7] R. Khalil, M. Al Horani, A. Yousef, and M. Sababheh, "A new definition of fractional derivative," Journal of Computational and Applied Mathematics, vol. 264, pp. 65-70, 2014.

[8] N. V. Hoa, H. Vu, and T. M. Duc, "Fuzzy fractional differential equations under Caputo-Katugampola fractional derivative approach," Fuzzy Sets and Systems, vol. 375, pp. 70-99, 2019.

[9] H. Y. Goo and J. S. Park, "On the continuity of the Zadeh extensions," Chungcheong Mathematical Society, vol. 20, no. 4, pp. 525-533, 2007.

[10] G. A. Anastassiou and S. G. Gal, "On a fuzzy trigonometric approximation theorem of Weierstrass-type," Journal of Fuzzy Mathematics, vol. 9, pp. 701-708, 2001.

[11] M. Mazandarani and M. Najariyan, "Type-2 fuzzy fractional derivatives," Communications in Nonlinear Science and $\mathrm{Nu}$ merical Simulation, vol. 19, no. 7, pp. 2354-2372, 2014.

[12] L. S. Chadli, A. Harir, and S. Melliani, "Fuzzy Euler differential equation," SOP Transactions on Applied Mathematics, vol. 2, no. 1, pp. 1-12, 2015.

[13] B. Bede, I. J. Rudas, and A. L. Bencsik, "First order linear fuzzy differential equations under generalized differentiability," Information Sciences, vol. 177, no. 7, pp. 1648-1662, 2007.

[14] A. Harir, S. Melliani, and L. S. Chadli, "Hybrid fuzzy differential equations," AIMS Mathematics, vol. 5, no. 1, pp. 273-285, 2019.

[15] A. Harir, S. Melliani, and L. S. Chadli, "Fuzzy fractional evolution equations and fuzzy solution operators," Advances in Fuzzy Systems, vol. 2019, Article ID 5734190, 10 pages, 2019. 